PROCEEDINGS OF THE

AMERICAN MATHEMATICAL SOCIETY

Volume 126, Number 9, September 1998, Pages 2763-2769

S 0002-9939(98)04659-0

\title{
NONSYMMETRIC OSSERMAN INDEFINITE KÄHLER MANIFOLDS
}

\author{
A. BONOME, R. CASTRO, E. GARCÍA-RÍO, L. HERVELlA, AND \\ R. VÁZQUEZ-LORENZO \\ (Communicated by Christopher Croke)
}

\begin{abstract}
The authors prove the existence of Osserman manifolds with indefinite Kähler metric of nonnegative or nonpositive holomorphic sectional curvature which are not locally symmetric.
\end{abstract}

\section{INTRODUCTION}

A semi-Riemannian manifold $(M, g)$ is said to be an Osserman space if the eigenvalues of the Jacobi operators $R_{X}$ are independent of the unit vectors $X$. Osserman conjectured that, in the Riemannian case, such spaces must be flat or locally isometric to a rank-one symmetric space [13]. This was proved for any $n^{-}$ dimensional Riemannian manifold with $n \neq 4 k(k>1)$ by Chi, who also showed that the Osserman conjecture holds for positive definite Kähler manifolds with nonpositive or nonnegative sectional curvature [5]. (See also [6] and [11] for related work and further references.) The study of the Osserman problem in Lorentzian geometry is, in some sense, simpler than in the Riemannian case, and a Lorentzian manifold is Osserman if and only if it is of constant curvature (see [2], [9]). In contrast to this case, in [10] the authors construct examples of semi-Riemannian Osserman spaces with metric of any signature $(p, q), p, q \geq 2$, which are not locally symmetric.

On the other hand, the holomorphic sectional curvature is a real function defined on the unit sphere bundle of any positive definite almost Hermitian manifold, and thus bounded at each point. However, for indefinite metrics such a statement is no longer true, and the holomorphic sectional curvature of an indefinite almost Hermitian manifold is bounded at a point if and only if it is constant at that point [1], [3]. Therefore, one could expect to obtain some affirmative conclusions on the study of the Osserman problem, as Chi did for positive definite Kähler metrics [5], by imposing some boundedness conditions on the holomorphic sectional curvature of an indefinite Kähler manifold. In this paper we will show that this is not the case by constructing examples of Osserman indefinite Kähler manifolds with nonnegative or

Received by the editors January 28, 1997.

1991 Mathematics Subject Classification. Primary 53B30, 53C15, 53C50, 53C55.

Key words and phrases. Osserman space, tangent bundle, indefinite Kähler metric, holomorphic sectional curvature, para-Kähler metric.

Supported by projects DGICYT PB940633C0201 and XUGA 20702B96, Spain.

(C)1998 American Mathematical Society 
nonpositive holomorphic sectional curvature which are not of constant holomorphic sectional curvature, even if they are locally symmetric.

The paper is organized as follows. In section 2 , we construct a broad family of semi-Riemannian Osserman manifolds by looking at the tangent bundle of certain semi-Riemannian manifolds equipped with a metric tensor related to the complete lift of that of the base manifold (cf. Theorem 1). $\S 3$ is devoted to analyzing the simplest case in Theorem 1, and we show that it provides a way to construct new examples of Osserman indefinite Kähler metrics whose holomorphic sectional curvature is nonnegative or nonpositive at each point (Theorem 4). Finally, since para-Hermitian geometry plays an important role in the study of semi-Riemannian metrics, we study such structures in $\S 4$ to show the existence of Osserman paraKähler manifolds with nonnegative or nonpositive paraholomorphic sectional curvature which are not paracomplex space forms.

\section{The DEFormed COMPlete LifT METRIC}

Let $T M$ denote the tangent bundle of an $n$-dimensional semi-Riemannian manifold $(M, g)$. This $2 n$-dimensional manifold may be equipped with the semi-Riemannian complete lift metric $g^{C}$, of signature $(n, n)$, defined by

$$
g^{C}\left(X^{C}, Y^{C}\right)=g(X, Y)^{C},
$$

where $X^{C}, Y^{C}$ are the complete lifts to $T M$ of the vector fields $X, Y$ on $M$. Although for a given semi-Riemannian metric $g$ on $M$ the projection $\pi:\left(T M, g^{C}\right)$ $\rightarrow(M, g)$ is not a semi-Riemannian submersion, $g^{C}$ reflects in a nice way some interesting properties of $(M, g)$. (We refer to [14] for more information.) As a generalization of the complete lift metric, we introduce the family of metrics on $T M$ we are interested in. Let $(M, g)$ be a semi-Riemannian manifold and $\phi$ a symmetric $(0,2)$-tensor field on $M$. On $T M$ we define the deformed complete lift metric $g_{\phi}$ of the semi-Riemannian metric $g$ by

$$
g_{\phi}=g^{C}+\phi^{V} .
$$

For local coordinates $\left(x^{1}, \ldots, x^{2 n}\right)=\left(x^{1}, \ldots, x^{n} ; x^{\overline{1}}, \ldots, x^{\bar{n}}\right)$ on $T M$ induced from coordinates $\left(x^{i}\right)$ on $M$, where $\bar{i}=i+n, i=1, \ldots, n$, we have the local expression

$$
g_{\phi}=\left(\begin{array}{cc}
\phi_{i j}+\frac{\partial g_{i j}}{\partial x^{k}} x^{\bar{k}} & g_{i j} \\
g_{i j} & 0
\end{array}\right),
$$

where $\left(\phi_{i j}\right)$ is the expression of $\phi$ in coordinates $(i, j=1, \ldots, n)$.

This kind of metrics were previously considered by Oproiu in the study of harmonic sections of the tangent bundle [12]. The purpose of this note is to show that they exhibit some exceptional behaviour from the point of view of the curvature, when comparing with the Riemannian case. We begin with the following

Theorem 1. Let $(M, g)$ be a semi-Riemannian manifold and $\phi$ any symmetric $(0,2)$-tensor field on $M$. Then, the tangent bundle $T M$ equipped with the deformed complete lift metric $g_{\phi}=g^{C}+\phi^{V}$ is an indefinite Osserman manifold if and only if $(M, g)$ is Osserman with zero eigenvalues of the Jacobi operator.

Proof. Let $\left(U,\left(x^{i}\right)\right)$ be a coordinate neighborhood on $M,\left(\pi^{-1}(U),\left(x^{i}, x^{\bar{i}}\right)\right)$ the induced coordinates on $T M$ and $\tilde{X}=\sum_{i=1}^{n}\left\{\alpha_{i} \frac{\partial}{\partial x^{i}}+\alpha_{\bar{i}} \frac{\partial}{\partial x^{i}}\right\}$ a vector field on $T M$, 
where $\left\{\frac{\partial}{\partial x^{i}}, \frac{\partial}{\partial x^{i}}\right\}$ is the local basis of vector fields on $T M$ given by the coordinate vector fields. After a straightforward calculation, one gets the following components $\tilde{R}_{\alpha \beta \gamma}^{\delta}$ of the curvature tensor $\tilde{R}$ of $\left(T M, g_{\phi}\right)$ :

$$
\begin{array}{r}
\tilde{R}_{i j \bar{k}}^{l}=\tilde{R}_{i \bar{j} k}^{l}=\tilde{R}_{\bar{i} \bar{j} k}^{l}=\tilde{R}_{i \bar{j} \bar{k}}^{l}=\tilde{R}_{\overline{i j} \bar{k}}^{l}=0, \\
\tilde{R}_{i j k}^{l}=\tilde{R}_{i j \bar{k}}^{\bar{l}}=\tilde{R}_{i \bar{j} k}^{\bar{l}}=R_{i j k}^{l}, \\
\tilde{R}_{\bar{i} \bar{l} k}^{\bar{l}}=\tilde{R}_{i \bar{j} \bar{k}}^{\bar{l}}=\tilde{R}_{\overline{i j k}}^{\bar{l}}=0,
\end{array}
$$

where $R_{i j k}^{l}$ are the components of the curvature tensor of $(M, g)$. Therefore, the matrix form of the Jacobi operator $\tilde{R}_{\tilde{X}}=\tilde{R}(\cdot, \tilde{X}) \tilde{X}$ at a point $\tilde{p} \in T M$ on the basis induced by the coordinate vector fields becomes

$$
\tilde{R}_{\tilde{X}}=\left(\begin{array}{cc}
R_{X} & 0 \\
* & R_{X}
\end{array}\right),
$$

where $X$ is the vector $X=\sum_{i=1}^{n} \alpha_{i} \frac{\partial}{\partial x^{i}}$ tangent to $M$ at $p=\pi(\tilde{p})$ and $R_{X}$ is the matrix of the Jacobi operator $R_{X}$ on the basis induced by the coordinate vector fields on $M$.

Next, let $X=\sum_{i=1}^{n} \alpha_{i} \frac{\partial}{\partial x^{i}}$ be a nonnull vector on $T_{p} M$ and take $\tilde{X}$ at $T_{\tilde{p}} T M$ with $\tilde{p}=(p, 0)$ given by $\tilde{X}=\sum_{i=1}^{n}\left\{\alpha_{i} \frac{\partial}{\partial x^{i}}+\alpha_{\bar{i}} \frac{\partial}{\partial x^{\bar{i}}}\right\}$, where the $\alpha_{\bar{i}}$ are defined as follows. Choose $k \in\{1, \ldots, n\}$ in such a way that $\sum_{i=1}^{n} \alpha_{i} g_{i k} \neq 0$ (note that this is always possible since $X$ is a nonnull vector) and define

$$
\alpha_{\bar{t}}=\left\{\begin{array}{cl}
\frac{1-\sum_{i, j=1}^{n} \alpha_{i} \alpha_{j} \phi_{i j}}{2 \sum_{i=1}^{n} \alpha_{i} g_{i k}} & \text { if } t=k, \\
0 & \text { if } t \neq k .
\end{array}\right.
$$

Then, since

$$
g_{\phi}(\tilde{X}, \tilde{X})=\sum_{i, j, r=1}^{n} \alpha_{i} \alpha_{j} x^{\bar{r}} \frac{\partial g_{i j}}{\partial x^{r}}+\sum_{i, j=1}^{n} \alpha_{i} \alpha_{j} \phi_{i j}+2 \sum_{i, j=1}^{n} \alpha_{i} \alpha_{\bar{j}} g_{i j}
$$

$\tilde{X}$ is a unit vector on the zero section of $T M$.

Now, if $\left(T M, g_{\phi}\right)$ is assumed to be Osserman, it follows from (4) that $(M, g)$ is also Osserman. Moreover, since the Jacobi operators $R_{X}$ must have constant eigenvalues independent of the nonnull vector $X$ on $M$, these eigenvalues are necessarily vanishing. Conversely, if $(M, g)$ is assumed to be Osserman with vanishing eigenvalues of the Jacobi operator, then it follows from (4) that the characteristic polynomial $p_{\lambda}\left(\tilde{R}_{\tilde{X}}\right)=\operatorname{det}\left(\tilde{R}_{\tilde{X}}-\lambda I_{2 n}\right)$ of the Jacobi operator $\tilde{R}_{\tilde{X}}$ satisfies $p_{\lambda}\left(\tilde{R}_{\tilde{X}}\right)$ $=\lambda^{2 n}$ for any vector $\tilde{X}$ tangent to $T M$. Hence $\left(T M, g_{\phi}\right)$ is Osserman.

Remark 2. A Riemannian or Lorentzian Osserman manifold with zero eigenvalues of the Jacobi operator is necessarily flat. This is no longer true, however, for 
higher signatures. Indeed, all the examples of Osserman semi-Riemannian manifolds constructed in [10] have zero eigenvalues of the Jacobi operator, but they are not flat. Also note that, if $(M, g)$ is a flat manifold, $\left(T M, g_{\phi}\right)$ is an Osserman semi-Riemannian manifold which is not necessarily flat (just consider $\phi$ to be any conformal change of the metric $g$ ).

\section{INDEFINITE KÄHLER MANIFOLDS}

In this section we will analyse the simplest case of Theorem 1 by studying the deformed complete lift metric of the usual Riemannian metric on $\mathbb{R}^{2}$. Therefore we will consider the 4 -dimensional space $\mathbb{R}^{4}$ with coordinates $\left(x^{1}, x^{2}, x^{3}, x^{4}\right)=$ $\left(x^{1}, x^{2}, x^{\overline{1}}, x^{\overline{2}}\right),\left(x^{1}, x^{2}\right)$ being the usual coordinates on $\mathbb{R}^{2}$. Now, let $\phi=\left(\phi_{i j}\right)$ be a symmetric $(0,2)$-tensor field on $\mathbb{R}^{2}$ and consider the deformed complete lift metric $g_{\phi}$ on $\mathbb{R}^{4}$, which is expressed by

$$
g_{\phi}=d x^{1} \otimes d x^{3}+d x^{2} \otimes d x^{4}+\sum_{i, j=1}^{2} \phi_{i j} d x^{i} \otimes d x^{j} .
$$

The Levi-Civita connection of $\left(\mathbb{R}^{4}, g_{\phi}\right)$ is given by

$$
\begin{aligned}
& \nabla_{\frac{\partial}{\partial x^{1}}} \frac{\partial}{\partial x^{1}}=\frac{1}{2} \frac{\partial \phi_{11}}{\partial x^{1}} \frac{\partial}{\partial x^{3}}+\left[-\frac{1}{2} \frac{\partial \phi_{11}}{\partial x^{2}}+\frac{\partial \phi_{12}}{\partial x^{1}}\right] \frac{\partial}{\partial x^{4}}, \\
& \nabla_{\frac{\partial}{\partial x^{1}}} \frac{\partial}{\partial x^{2}}=\frac{1}{2} \frac{\partial \phi_{11}}{\partial x^{2}} \frac{\partial}{\partial x^{3}}+\frac{1}{2} \frac{\partial \phi_{22}}{\partial x^{1}} \frac{\partial}{\partial x^{4}}, \\
& \nabla_{\frac{\partial}{\partial x^{2}}} \frac{\partial}{\partial x^{2}}=\left[-\frac{1}{2} \frac{\partial \phi_{22}}{\partial x^{1}}+\frac{\partial \phi_{12}}{\partial x^{2}}\right] \frac{\partial}{\partial x^{3}}+\frac{1}{2} \frac{\partial \phi_{22}}{\partial x^{2}} \frac{\partial}{\partial x^{4}},
\end{aligned}
$$

and hence the only nonvanishing components of the curvature tensor are

$$
\begin{aligned}
& R\left(\frac{\partial}{\partial x^{1}}, \frac{\partial}{\partial x^{2}}\right) \frac{\partial}{\partial x^{1}}=\left[\frac{1}{2} \frac{\partial^{2} \phi_{11}}{\partial x^{2} \partial x^{2}}+\frac{1}{2} \frac{\partial^{2} \phi_{22}}{\partial x^{1} \partial x^{1}}-\frac{\partial^{2} \phi_{12}}{\partial x^{1} \partial x^{2}}\right] \frac{\partial}{\partial x^{4}}, \\
& R\left(\frac{\partial}{\partial x^{1}}, \frac{\partial}{\partial x^{2}}\right) \frac{\partial}{\partial x^{2}}=-\left[\frac{1}{2} \frac{\partial^{2} \phi_{11}}{\partial x^{2} \partial x^{2}}+\frac{1}{2} \frac{\partial^{2} \phi_{22}}{\partial x^{1} \partial x^{1}}-\frac{\partial^{2} \phi_{12}}{\partial x^{1} \partial x^{2}}\right] \frac{\partial}{\partial x^{3}} .
\end{aligned}
$$

Then, we have

Theorem 3. $\left(\mathbb{R}^{4}, g_{\phi}\right)$ is a semi-Riemannian Osserman manifold. Moreover, it is locally symmetric if and only if

$$
\frac{1}{2} \frac{\partial^{2} \phi_{11}}{\partial x^{2} \partial x^{2}}+\frac{1}{2} \frac{\partial^{2} \phi_{22}}{\partial x^{1} \partial x^{1}}-\frac{\partial^{2} \phi_{12}}{\partial x^{1} \partial x^{2}}
$$

is a constant function, where $\phi_{i j}, i, j=1,2$, are the components of any symmetric $(0,2)$-tensor field on $\mathbb{R}^{2}$.

Proof. Clearly $\left(\mathbb{R}^{4}, g_{\phi}\right)$ is an Osserman space as an application of Theorem 1. Now the second part follows after a straightforward calculation from (6) and (7).

Next, let $J$ denote the usual complex structure on $\mathbb{R}^{2}\left(J \frac{\partial}{\partial x^{1}}=\frac{\partial}{\partial x^{2}}\right)$ and let $J^{C}$ be its complete lift to $\mathbb{R}^{4}=T \mathbb{R}^{2}$. A symmetric $(0,2)$-tensor field $\phi$ on $\left(\mathbb{R}^{2}, g, J\right)$ is called Hermitian if it satisfies $\phi(J X, J Y)=\phi(X, Y)$ for all vector fields on $\mathbb{R}^{2}$. (Equivalently, $\phi_{11}=\phi_{22}$ and $\phi_{12}=0$.) Now, it follows from (5) that $g_{\phi}$ is an indefinite almost Hermitian metric on $\left(\mathbb{R}^{4}, J^{C}\right)$ if and only if $\phi$ is Hermitian. 
Theorem 4. Let $\phi$ be an Hermitian $(0,2)$-tensor field on $\left(\mathbb{R}^{2}, g, J\right)$. Then $\left(\mathbb{R}^{4}, g_{\phi}, J^{C}\right)$ is an Osserman indefinite Kähler manifold, and, moreover, the holomorphic sectional curvature of $\left(\mathbb{R}^{4}, g_{\phi}, J^{C}\right)$ has the sign of $\Delta \phi_{11}$, where $\Delta$ denotes the Laplacian on $\mathbb{R}^{2}$.

Proof. Since $\phi$ is Hermitian, $\phi_{11}=\phi_{22}$ and $\phi_{12}=0$. Then it follows from (6) after a straightforward calculation that $\left(\mathbb{R}^{4}, g_{\phi}, J^{C}\right)$ is an indefinite Kähler manifold. Moreover, the expression (7) of the curvature tensor becomes

$$
\begin{aligned}
& R\left(\frac{\partial}{\partial x^{1}}, \frac{\partial}{\partial x^{2}}\right) \frac{\partial}{\partial x^{1}}=\frac{1}{2} \Delta \phi_{11} \frac{\partial}{\partial x^{4}} \\
& R\left(\frac{\partial}{\partial x^{1}}, \frac{\partial}{\partial x^{2}}\right) \frac{\partial}{\partial x^{2}}=-\frac{1}{2} \Delta \phi_{11} \frac{\partial}{\partial x^{3}}
\end{aligned}
$$

where $\Delta=\frac{\partial^{2}}{\partial x^{1} \partial x^{1}}+\frac{\partial^{2}}{\partial x^{2} \partial x^{2}}$ is the Euclidean Laplacian on $\mathbb{R}^{2}$. Thus, the holomorphic sectional curvature of any holomorphic nondegenerate plane $\{X, J X\}$ is given by

$$
H(X)=\frac{g_{\phi}(R(X, J X) X, J X)}{g_{\phi}(X, X)^{2}}=\frac{1}{2}\left(\frac{\alpha_{1}^{2}+\alpha_{2}^{2}}{g_{\phi}(X, X)}\right)^{2} \Delta \phi_{11}
$$

where $X$ is a vector field $X=\sum \alpha_{i} \frac{\partial}{\partial x^{i}}$. This shows that the holomorphic sectional curvature takes the sign of $\Delta \phi_{11}$.

Corollary 5. If $\left(\mathbb{R}^{4}, g_{\phi}, J^{C}\right)$ is a locally symmetric Osserman indefinite Kähler manifold, then the holomorphic sectional curvature of $\left(\mathbb{R}^{4}, g_{\phi}, J^{C}\right)$ is nonpositive or nonnegative, but not constant unless it is flat.

Proof. Note that, if $\phi$ is an Hermitian (0,2)-tensor field on $\mathbb{R}^{2}$, then (8) is reduced to $\Delta \phi_{11}$, and the result follows.

We finish this section with some remarks.

Remark 6. Note that the product of the manifolds $\left(\mathbb{R}^{4}, g_{\phi}, J^{C}\right)$ and the Euclidean spaces $\mathbb{R}_{\nu}^{n}$ allows us to construct indefinite Kähler manifolds of any signature $(2 p, 2 q), p, q \geq 1$, with nonnegative or nonpositive holomorphic sectional curvature, which are Osserman but not locally symmetric.

Remark 7. We recall here that at each point of $\left(\mathbb{R}^{4}, g_{\phi}, J^{C}\right)$ the holomorphic sectional curvature is nonnegative or nonpositive, although its sign may change from point to point as $\Delta \phi_{11}$ does.

Other kinds of boundedness conditions on the holomorphic sectional curvature of indefinite almost Hermitian manifolds have been investigated in [4], where it is proved that if the holomorphic sectional curvature of an indefinite Kähler manifold is bounded from below (or from above) on holomorphic planes of signature $(+,+)$ and from above (or from below) on holomorphic planes of signature $(-,-)$, then the manifold is null-holomorphically flat. Such a condition, together with the Osserman one, immediately implies the constancy of the holomorphic sectional curvature, since any Osserman manifold is Einstein. 


\section{PARA-KähleR EXAMPleS}

Para-Kähler manifolds are symplectic manifolds locally diffeomorphic to a product of Lagrangian submanifolds. Equivalently, a para-Kähler manifold is a triple $(M, h, K)$, where $K$ is a paracomplex structure $\left(K^{2}=\mathrm{id}\right), h$ is a semi-Riemannian metric on $M$ such that

$$
h(K X, K Y)=-h(X, Y)
$$

for all vector fields $X, Y$ on $M$, and, moreover, $\nabla K=0$, where $\nabla$ is the Levi-Civita connection of $h$. We refer to [8] for the definition of the paraholomorphic sectional curvature, and to [7] for a survey on paracomplex geometry and further references.

Next consider the usual Minkowski metric $h$ on $\mathbb{R}_{1}^{2}$ and its deformed complete lift metric induced by a symmetric $(0,2)$-tensor field $\phi$ on the tangent bundle $\mathbb{R}^{4}$ $=T \mathbb{R}^{2}$ :

$$
h_{\phi}=d x^{1} \otimes d x^{3}-d x^{2} \otimes d x^{4}+\sum_{i, j=1}^{2} \phi_{i j} d x^{i} \otimes d x^{j} .
$$

$\left(\mathbb{R}^{2}, h\right)$ can be naturally endowed with a para-Kähler structure, just considering the paracomplex structure $K$ defined by $K \frac{\partial}{\partial x^{1}}=\frac{\partial}{\partial x^{2}}$. Now, we will say that a $(0,2)$-tensor field $\phi$ on $\mathbb{R}_{1}^{2}$ is para-Hermitian if it satisfies $\phi(K X, K Y)=-\phi(X, Y)$ for all vector fields on $\mathbb{R}_{1}^{2}$. (Equivalently, $\phi_{11}=-\phi_{22}$ and $\phi_{12}=0$.) It is now immediate to recognize from $(9)$ that $h_{\phi}$ is an almost para-Hermitian metric on $\left(\mathbb{R}^{4}, K^{C}\right)$ if and only if $\phi$ is para-Hermitian.

Theorem 8. Let $\phi$ be a para-Hermitian tensor on $\left(\mathbb{R}^{2}, h, K\right)$. Then $\left(\mathbb{R}^{4}, h_{\phi}, K^{C}\right)$ is an Osserman para-Kähler manifold; moreover, the paraholomorphic sectional curvature of $\left(\mathbb{R}^{4}, h_{\phi}, K^{C}\right)$ has the sign of $\square \phi_{11}$, where $\square$ denotes the hyperbolic Laplacian on $\mathbb{R}_{1}^{2}$.

Proof. The expressions of the Levi-Civita connection and the curvature tensor of (9) are similar to (6) and (7). Now, proceeding in a way similar to that of Theorem 4 , we find that $\left(\mathbb{R}^{4}, h_{\phi}, K^{C}\right)$ is an Osserman para-Kähler manifold. Moreover, the only nonvanishing components of the curvature tensor are those given by

$$
\begin{aligned}
& R\left(\frac{\partial}{\partial x^{1}}, \frac{\partial}{\partial x^{2}}\right) \frac{\partial}{\partial x^{1}}=\frac{1}{2} \square \phi_{11} \frac{\partial}{\partial x^{4}}, \\
& R\left(\frac{\partial}{\partial x^{1}}, \frac{\partial}{\partial x^{2}}\right) \frac{\partial}{\partial x^{2}}=\frac{1}{2} \square \phi_{11} \frac{\partial}{\partial x^{3}}
\end{aligned}
$$

where $\square=\frac{\partial^{2}}{\partial x^{1} \partial x^{1}}-\frac{\partial^{2}}{\partial x^{2} \partial x^{2}}$ is the hyperbolic Laplacian on $\mathbb{R}_{1}^{2}$. Hence, the paraholomorphic sectional curvature of any nondegenerate paraholomorphic plane $\{X, K X\}$ is given by

$$
H(X)=\frac{h_{\phi}(R(X, K X) X, K X)}{h_{\phi}(X, X) h_{\phi}(K X, K X)}=\frac{1}{2}\left(\frac{\alpha_{1}^{2}-\alpha_{2}^{2}}{h_{\phi}(X, X)}\right)^{2} \square \phi_{11},
$$

where $X=\sum \alpha_{i} \frac{\partial}{\partial x^{i}}$. This shows that the holomorphic sectional curvature takes the sign of $\square \phi_{11}$. 
Remark 9. The deformed complete lift metric of the Minkowski metric on $\mathbb{R}_{1}^{2}$ is locally symmetric if and only if

$$
\frac{1}{2} \frac{\partial^{2} \phi_{11}}{\partial x^{2} \partial x^{2}}+\frac{1}{2} \frac{\partial^{2} \phi_{22}}{\partial x^{1} \partial x^{1}}-\frac{\partial^{2} \phi_{12}}{\partial x^{1} \partial x^{2}}
$$

is a constant function. This reduces to $\square \phi_{11}=$ const when $\phi$ is a para-Hermitian tensor on $\mathbb{R}_{1}^{2}$.

Remark 10. As well as for indefinite Kähler manifolds, the paraholomorphic sectional curvature of a para-Kähler manifold is bounded from above and from below if and only if it is constant. However, it may be bounded from above or from below without being constant even if it is locally symmetric, as we see from Theorem 8 .

\section{REFERENCES}

[1] M. Barros and A. Romero, Indefinite Kähler manifolds, Math. Ann. 261 (1982), 55-62. MR 84d:53033

[2] N. Blažić, N. Bokan and P. Gilkey, A Note on Osserman Lorentzian manifolds, Bull. London Math. Soc. 29 (1997), 227-230. MR 97m:53111

[3] A. Bonome, R. Castro, E. García-Río and L. Hervella, On the holomorphic sectional curvature of an indefinite Kähler manifold, C. R. Acad. Sci. Paris 315 (1992), 1183-1187. MR 93j:53079

[4] A. Bonome, R. Castro, E. García-Río, L. Hervella and Y. Matsushita, Null holomorphically flat indefinite almost Hermitian manifolds, Illinois J. Math. 39 (1995), 635-660. MR 97e:53050

[5] Q.S. Chi, A curvature characterization of certain locally rank-one symmetric spaces, J. Diff. Geom. 28 (1988), 187-202. MR 90a:53060

[6] Q.S. Chi, Curvature characterization and classification of rank-one symmetric spaces, Pacific J. Math. 150 (1991), 31-42. MR 92g:53044

[7] V. Cruceanu, P. Fortuny and P.M. Gadea, A survey on paracomplex geometry, Rocky Mountain J. Math. 26 (1996), 83-115. MR 97c:53112

[8] P.M. Gadea and A. Montesinos Amilibia, Spaces of constant paraholomorphic sectional curvature, Pacific J. Math. 136 (1989), 85-101. MR 90d:53043

[9] E. García-Río, D.N. Kupeli and M.E. Vázquez-Abal, On a problem of Osserman in Lorentzian geometry, Diff. Geom. Appl. 7 (1997), 85-100. CMP 97:10

[10] E. García-Río, M.E. Vázquez-Abal and R. Vázquez-Lorenzo, Nonsymmetric Osserman pseudo-Riemannian manifolds, preprint.

[11] P. Gilkey, A. Swann and L. Vanhecke, Isoparametric geodesic spheres and a conjecture of Osserman concerning the Jacobi operator, Quart. J. Math. Oxford 46 (1995), 299-320. MR 96h:53051

[12] V. Oproiu, Harmonic maps between tangent bundles, Rend. Sem. Mat. Univ. Politecn. Torino 47 (1989), 47-55. MR 92f:58047

[13] R. Osserman, Curvature in the eighties, Amer. Math. Monthly 97 (1990), 731-756. MR 91i:53001

[14] K. Yano, S. Ishihara, Tangent and cotangent bundles, Marcel Dekker, New York, 1973. MR 50:3142

Facultade de Matemáticas, Universidade de Santiago de Compostela, 15706 Santiago de Compostela, Spain

E-mail address, E. García-Río: eduardo@zmat.usc.es 